\title{
Actualización de Métodos de Enseñanza-Aprendizaje en Asignaturas de Dirección de Proyectos de Ingeniería
}

\author{
José M. Mesa, José V. Álvarez, Joaquín M. Villanueva y Francisco J. de Cos \\ Universidad de Oviedo, Escuela Politécnica Superior de Ingeniería de Gijón, Área de \\ Proyectos de Ingeniería, Carretera de Villaviciosa s/n, 33203 Gijón, Asturias-España \\ (email: jmmesa@uniovi.es)
}

\section{Resumen}

Se analiza la implementación de la metodología ABP (Aprendizaje Basado en Problemas o Proyectos) en asignaturas de Dirección de Proyectos de Ingeniería. La aplicación de la metodología ABP con el uso de las nuevas tecnologías de la información y la comunicación (TIC) permite actualizar el aprendizaje de los alumnos, acercándolo al desempeño de su futuro laboral. Se describe la experiencia llevada a cabo en la docencia de la asignatura "Proyectos" impartida en la titulación Ingeniería Industrial de la Escuela Politécnica Superior de Ingeniería de Gijón (EPSIG) de la Universidad de Oviedo, España. Se discuten los resultados obtenidos en base a las aportaciones tanto de los alumnos como del equipo docente. La metodología ABP resulta muy adecuada para el aprendizaje de competencias en el ámbito de la ingeniería, pero exige una definición precisa de objetivos y una adecuada planificación, así como el seguimiento y evaluación continuos del profesor.

\section{An Update of Teaching/Learning Methods in Project Management Courses}

\begin{abstract}
The implementation of the PBL methodology (Problem or Project Based Learning) in Project Management courses in an engineering degree is analyzed. The PBL methodology that includes new information and communication technologies (ICT), allows updating the learning of the students approaching them to their future professional work. The study-case analyzed is the course "Projects" given for the Industrial Engineering degree of the Higher Polytechnic School of Engineering of Gijón (EPSIG), which belongs to the University of Oviedo in Spain. The results obtained are analyzed according to contributions by both the teaching staff and the students. The PBL methodology is found to be very appropriate for the learning of engineering competences, but it demands a precise definition of objectives and a detailed planning, as well as continuous monitoring and evaluation by the lecturer.
\end{abstract}

Keywords: higher education, project management, professional skills, PBL 


\section{INTRODUCCIÓN}

Actualmente la formación universitaria tiende a fomentar un aprendizaje más centrado en el alumno, creando un entorno más interactivo y motivador tanto para los alumnos como para los propios docentes. En las universidades públicas europeas dicha tendencia se ha visto impulsada por la denominada Declaración de Bolonia (EME, 1999) y la construcción del Espacio Europeo de Educación Superior (EEES). Entre sus principales objetivos está, además de homogeneizar las titulaciones, el acercar el aprendizaje en las competencias profesionales. Este concepto pretende mejorar la relación del sistema educativo con el productivo, con el objetivo de impulsar una adecuada formación de los profesionales. Teniendo en cuenta esta idea, el paso previo a la planificación de una asignatura o materia debería consistir en el análisis de funciones y tareas que llevan a cabo los profesionales en ejercicio.

En el caso de la gestión o dirección de proyectos la importancia creciente en las distintas disciplinas de la ingeniería y de muchas de las tareas que supone (organización, planificación, presupuestación, gestión de recursos humanos, comunicación, control,...) ha sido resaltada en numerosas publicaciones (Palmer, 2003; Pulko y Parikh, 2003). Entidades como Project Management Institute (PMI) o la International Project Management Association (IPMA), han elaborado sendas Guías sobre los Fundamentos de la Dirección de Proyectos (IPMA, 2006; PMI, 2004) las cuales permiten establecer una buena referencia de las competencias profesionales del director de proyectos.

Según dichas publicaciones, la dirección de proyectos es "la aplicación de conocimientos, aptitudes, herramientas y técnicas a las actividades de proyecto, encaminados a satisfacer 0 colmar las necesidades y expectativas de una organización mediante un proyecto". Es decir, se trata fundamentalmente de equilibrar las demandas de alcance, plazos, coste y calidad de todas las entidades involucradas en el proyecto y sería, de forma resumida, el objetivo global del trabajo de un ingeniero de proyectos.

Teniendo en cuenta esa realidad social y académica, la revisión o actualización del proceso enseñanza-aprendizaje de la dirección o gestión de proyectos se describirá mediante su aplicación en la asignatura denominada "Proyectos". Dicha asignatura se imparte en el $5^{\circ}$ curso de la titulación de Ingeniería Industrial de la Escuela Politécnica Superior de Ingeniería de Gijón (EPSIG) perteneciente a Universidad de Oviedo (España). Inicialmente se realizó una revisión o determinación de los objetivos de aprendizaje para una mayor adaptación a las necesidades profesionales de los estudiantes en su futuro trabajo como ingenieros de proyectos identificando sus competencias profesionales a través de las Guías anteriormente mencionadas.

En la siguiente etapa se seleccionó la metodología colaborativa más adecuada para la consecución de dichos objetivos. La aplicación de la misma permitió ajustar progresivamente determinados elementos como los enunciados, el contenido de los entregables, el calendario o programación de las prácticas, el número o composición de los grupos, entre otros y siempre bajo la perspectiva de una mejora continua de la docencia.

Finalmente se incorporaron al proceso de aprendizaje las posibilidades ofrecidas por las nuevas tecnologías, teniendo en cuenta que éstas deben integrarse perfectamente en las actividades previstas para no distorsionar su realización y que los alumnos no pierdan de vista que el objetivo es el proyecto de ingeniería y no únicamente el uso de una determinada herramienta software.

\section{Objetivos de aprendizaje}

Teniendo en cuenta las referencias anteriores se enuncian a continuación las competencias que se han determinado para la asignatura "Proyectos". El propósito final es que los estudiantes de ingeniería sean capaces de plantear, realizar y dirigir proyectos, mediante la aplicación de los conocimientos científicos y técnicos necesarios. 
Tabla 1: Competencias genéricas o transversales para el perfil de ingeniería

\begin{tabular}{|l|}
\hline Desarrollo de destreza en la búsqueda, selección, análisis y evaluación de la información. \\
\hline $\begin{array}{l}\text { Adquisición de habilidades para las relaciones interpersonales y para el trabajo en grupo. Cooperación } \\
\text { para lograr un objetivo único. }\end{array}$ \\
\hline Adquirir una capacidad creativa e innovadora. \\
\hline Desarrollar la capacidad para enfrentarse a situaciones ambiguas como en la vida real. \\
\hline Generar alternativas en el proceso de toma de decisiones, argumentar y defender posicionamientos. \\
\hline Analizar y evaluar las alternativas generadas. \\
\hline Adquirir habilidades para la comunicación, presentación y argumentación de ideas y soluciones. \\
\hline
\end{tabular}

Es posible observar que una parte considerable de los objetivos de aprendizaje de esta disciplina se corresponden con competencias actualmente denominadas transversales (Tabla 1), que abarcan ámbitos amplios de la educación, como por ejemplo, el trabajo en equipo, la capacidad de análisis crítico y de comunicación. En la Tabla 2 se revisan las propias o específicas de la dirección de proyectos. Estas últimas se han clasificado en tres categorías según incidan en aspectos técnicos, humanos y de gestión.

\section{Tabla 2: Competencias específicas}

\begin{tabular}{l}
\hline \multicolumn{1}{|c|}{ Aspectos técnicos } \\
\hline Conocer las etapas del ciclo de vida de un proyecto de ingeniería, su contenido y sus entradas y salidas. \\
\hline $\begin{array}{l}\text { Aplicar conocimientos tecnológicos y científicos de forma sistemática y eficiente, integrando distintas } \\
\text { disciplinas, para alcanzar soluciones de ingeniería factibles. }\end{array}$ \\
\hline Desarrollar la posible implementación en función de la decisión adoptada. \\
\hline $\begin{array}{l}\text { Aprender a identificar, analizar y valorar los distintos factores técnicos, económicos, ambientales, } \\
\text { organizativos y sociales relacionados con el proyecto. }\end{array}$ \\
\hline Adquirir una capacidad creativa e innovadora. \\
\hline \multicolumn{1}{c|}{ Aspectos humanos } \\
\hline Identificar, valorar y considerar los factores humanos que afectan y son afectados por el proyecto. \\
\hline Desarrollar aptitudes para el trabajo en grupo formando parte de equipos multidisciplinares. \\
\hline Adquirir habilidades para la comunicación, presentación y argumentación de ideas y soluciones. \\
\hline \multicolumn{1}{c|}{ Aspectos de gestion o dirección } \\
\hline $\begin{array}{l}\text { Elaborar proyectos, anteproyectos, informes y demás documentación relacionada con el diseño y la } \\
\text { dirección de proyectos. }\end{array}$ \\
\hline $\begin{array}{l}\text { Afrontar procesos de toma de decisiones mediante la utilización de todos los recursos disponibles como la } \\
\text { búsqueda de información, la creatividad, la aplicación de metodologías y el diseño de soluciones. }\end{array}$ \\
\hline Aplicar técnicas de dirección de proyectos con el fin de planificar y controlar la ejecución del mismo. \\
\hline Gestionar las compras y contratación de recursos materiales y humanos. \\
\hline Manejar herramientas informáticas relacionadas con la gestión de proyectos. \\
\hline $\begin{array}{l}\text { Conocer las organizaciones y agentes que intervienen durante el ciclo de vida del proyecto, así como sus } \\
\text { funciones y responsabilidades. }\end{array}$ \\
\hline Conocer las funciones y responsabilidades del director de proyecto. \\
\hline $\begin{array}{l}\text { Tomar conciencia de la responsabilidad ética y legal ligada al ejercicio profesional en proyectos de } \\
\text { ingeniería, especialmente importante en la fase de ejecución de los mismos. }\end{array}$ \\
\hline
\end{tabular}

Resulta fundamental la determinación inicial de los objetivos de aprendizaje para plantear el rediseño de la asignatura y en la aplicación posterior de una metodología docente adecuada. Se puede observar que gran parte de esas competencias se refieren más a aspectos de habilidades prácticas del desempeño que a conocimientos o contenidos teóricos, lo que debe tener un reflejo en la metodología a emplear.

\section{Metodología}

En el esquema docente con la metodología anterior más tradicional, la enseñanza de esos conocimientos y competencias se realizaba anteriormente a través de clases teóricas, en las que el alumno tenía una escasa participación. El empleo una metodología de Aprendizaje Basado en 
Proyectos (también denominado PBL- Problem Based Learning), ya ha sido ampliamente documentada en diversas publicaciones (De Graaff y Kolmos, 2003), y permite centrar el aprendizaje en el alumno e introducir en la enseñanza problemas abiertos y más próximos al desempeño profesional. De esta forma el alumno adquiere un conjunto de competencias o habilidades que coinciden en gran medida con las competencias genéricas de la dirección de proyectos expuestas en la Tabla 1. El ABP es habitualmente empleado en la enseñanza de ingeniería, en gran variedad de disciplinas y con buenos resultados (Butun, 2005; Chinowsky et al., 2006; Johnson, 1999; Marti et al.,2006; Ribeiro y Mizukami, 2005). En algunos casos se ha indicado que el nivel alcanzado de conocimientos de carácter más técnico o teórico disminuyen con respecto a la orientación tradicional, aunque se incrementan en otra serie de habilidades o competencias (Schilling y Hagen, 2000). Por tanto se considera que la metodología que más se adapta a los objetivos de la asignatura es la combinación el empleo del ABP a través de la realización en equipo de un proyecto de ingeniería con algunas clases expositivas que se irán intercalando en el desarrollo del mismo.

\section{Incorporación de las TIC}

De la misma forma que el trabajo en las empresas de ingeniería ha evolucionado e incorpora cada día nuevas herramientas y modos de trabajo, la actividad docente debe adaptarse para aprovechar las posibilidades que las nuevas tecnologías ofrecen. En ese sentido son numerosas las experiencias docentes que están utilizando plataformas educativas como WebCT o Moodle para mejorar la calidad de la docencia en la educación superior (Manning, 2004; Moreno et al., 2007). En este caso se prefirió el empleó de una herramienta específica de gestión de proyectos (Microsoft Sharepoint) para favorecer el acercamiento de la acción docente al entorno profesional real.

La incorporación de las TIC al desarrollo del Aprendizaje Basado en Proyectos supone un salto cualitativo acercando la realización de las prácticas al desempeño profesional actual del ingeniero de proyectos. Resuelve además distintos problemas como el almacenamiento, intercambio, versiones de documentos y acceso a la información del proyecto de los grupos de alumnos. También facilita herramientas para la coordinación interna y la planificación, eliminando la necesidad de crear grupos en sitios Web como Yahoo o Google, poco adaptados a las necesidades docentes y que no permiten al profesor-tutor un seguimiento de las actividades de los alumnos.

El propio manejo de la herramienta empleada en proyecto constituye un objetivo de aprendizaje de las asignaturas puesto que este tipo de aplicaciones son habitualmente empleadas para la Gestión de Proyectos en distintas entidades y organizaciones, tanto públicas como privadas.

\section{APLICACIÓN METODOLÓGICA}

En el momento de diseñar una actividad de trabajo en grupo, como en este caso para la realización de un proyecto de ingeniería, fue importante considerar numerosos aspectos. Para que el grupo funcione adecuadamente todos los miembros son necesarios y deben trabajar de forma coordinada. Cada integrante debe tener presente que el resultado global del proyecto le afecta de forma individual, es decir, no sólo se le evalúa por la calidad de la parte en la que ha trabajado directamente. Es conveniente también que el profesor-tutor fomente la interacción y el análisis en común en el marco de las reuniones de seguimiento y coordinación, de las distintas alternativas, decisiones y problemas que se afrontarán dentro del grupo en el desarrollo del proyecto. El profesor-tutor debe estar preparado para enfrentarse a posibles conflictos internos y establecer desde la primera sesión algunas reglas básicas de funcionamiento y mecanismos para la toma de decisiones en caso de discrepancias, etc. El trabajo en equipo hizo necesario además el empleo de unos métodos de evaluación distintos a los empleados con una metodología tradicional (Palmer, 2000). Al término del proyecto el grupo evaluó y analizó junto al profesor-tutor el resultado del trabajo, con el fin de poner de relieve tanto aquellos aspectos que se que se deberían haber mejorado como aquellos que se realizaron con éxito. 
La aplicación del ABP en este caso se realizó encargando el desarrollo, presentación y defensa del mismo proyecto de ingeniería a los 8 grupos existentes, cada uno de ellos formado por entre 15 y 20 alumnos, lo que introducía ciertos elementos de competencia entre ellos. Dicho proyecto se llevó a cabo desde la concepción de la idea, la evaluación de su viabilidad desde los distintos puntos de vista técnico, económico, medioambiental, entre otros, y posteriormente su diseño, cálculo y documentación hasta su fase de contratación. Al final cada grupo debía presentar la documentación de su solución y realizar una exposición pública, con una duración entre 40-50 minutos frente a un tribunal de evaluación que realiza un conjunto de preguntas. La Guía de prácticas proporcionada a los alumnos enunciaba los objetivos de aprendizaje fijados para la práctica, el calendario de reuniones y entregables, la metodología y dinámica de la práctica, así como los criterios de evaluación y los recursos disponibles.

Aunque esta metodología supone que el alumno va a realizar una gran parte de su trabajo de forma autónoma, la coordinación del grupo, la corrección de desviaciones, detección de problemas de aprendizaje y finalmente, la necesidad de realizar una evaluación de los trabajos exigen una considerable labor de seguimiento por parte del profesor-tutor. Éste actuó además como representante de la empresa que promovió el proyecto.

El seguimiento del progreso del trabajo se realizó mediante la realización de reuniones semanales que permitieron al profesor-tutor realizar un seguimiento individualizado del trabajo del alumno, y no solo del avance global del grupo de prácticas. Por otro lado, se plantearon también algunas reuniones de carácter especial, por ejemplo, para el lanzamiento del proyecto y la definición de determinados roles (como el de director del proyecto, el encargado de planificar los trabajos), la preparación o ensayo de la presentación final y finalmente la de cierre de proyecto con la revisión de las lecciones aprendidas.

La evaluación del trabajo realizado, se hizo en base a la documentación y la presentación oral, que fueron juzgados por un tribunal de profesores, así como de los datos obtenidos durante el seguimiento semanal por parte del tutor de grupo.

\section{CONCLUSIONES}

La metodología de Aprendizaje Basado en Proyectos (ABP) resultó muy adecuada para el aprendizaje de competencias en el ámbito de la ingeniería. En su aplicación resultó muy importante delimitar adecuadamente los objetivos de aprendizaje y el alcance del proyecto propuesto, lo que permitió estimar adecuadamente el trabajo no presencial requerido, de forma que no superó lo esperado. En este sentido fue muy importante el trabajo en equipo de los profesores de la asignatura para definir perfectamente el contenido y el grado de complejidad a alcanzar.

La realización del proyecto de prácticas en el ABP supone para el alumno un considerable número de horas de trabajo fuera del aula. En el seguimiento realizado en este caso estaba alrededor del 55 por ciento y se dedicaba fundamentalmente a las tareas de búsqueda de información, análisis y diseño de soluciones y elaboración de documentación. La obtención de datos del tiempo de dedicación de los alumnos al proyecto resultó muy útil al tutor para valorar su trabajo y le permitió contrastar lo observado durante las reuniones de seguimiento.

La incorporación de las nuevas tecnologías facilitó el trabajo colaborativo de los alumnos y las tareas de seguimiento y evaluación de los docentes.

Se ha detectado su valor en algunas carencias en la preparación de los alumnos para enfrentarse al análisis, valoración y selección de alternativas que les permita realizar una toma de decisiones fundamentada. Esto se debe fundamentalmente a que están habituados a resolver problemas cerrados, con una solución única y no a tomar decisiones, algo fundamental en su futuro trabajo, en este caso como ingenieros. 
Por otro lado, los alumnos están poco acostumbrados a trabajar en equipo. De forma general funcionan mejor los grupos relativamente pequeños (entre 10 y 15 alumnos) donde cada estudiante percibe en su trabajo una clara dependencia del trabajo de los demás, lo que ayuda a cohesionar el grupo. Sin embargo en grupos mayores el trabajo de cada alumno se difumina con mayor facilidad. Salvo algunas excepciones, la mayoría de los alumnos se integraron adecuadamente dentro de sus grupos y percibieron como positiva la metodología empleada en la asignatura aunque les suponía una elevada carga de trabajo.

Finalmente se debe insistir en la necesidad de mejorar las fuentes de información, actualización de bibliografía, recopilación de normas, reglamentos, etc. de forma que Internet no sea la principal fuente de la misma. También sería conveniente que los alumnos dispusieran de más documentos de ejemplo que les proporcionaran una referencia metodológica para su trabajo.

\section{REFERENCIAS}

Butun, E.; Teaching genetic algorithms in electrical engineering education: a problem-based learning approach, International Journal of Electrical Engineering Education: 42(3), 223-233 (2005)

Chinowsky, P.S., H. Brown, A. Szajnman y A. Realph; Developing knowledge landscapes through project-based learning, Journal of Professional Issues in Engineering Education and Practice: 132(2), 118-124 (2006)

de Graaff, E. y A. Kolmos; Characteristics of problem-based learning, International Journal of Engineering Education: 19(5), 657-662 (2003)

EME; Joint declaration of the European Ministers of Education; Bologna Declaration (1999)

IPMA; International Project Management Association, ICB - IPMA Competence Baseline, ISBN: 09553213-0-1 (2006)

Johnson, P.A.; Problem-based, cooperative learning in the engineering classroom, Journal of Professional Issues in Engineering Education and Practice: 125(1), 8-11 (1999)

Manning, C.K.; WebCT discussions in problem based learning (PBL): analysis of use by representative groups in an introductory food science course, Journal of the American Dietetic Association: 104(Supplement 2), 40-341 (2004)

Marti, E., D. Gil y C. Julia; A PBL experience in the teaching of computer graphics, Computer Graphics Forum: 25(1), 95-103 (2006)

Moreno, Lorenzo y otros cuatro autores; Applying a constructivist and collaborative methodological approach in engineering education, Computers \& Education: 49(3), 891-915 (2007)

Palmer, S.R.; Student responses to activities designed to develop generic professional skills, Journal of Professional Issues in Engineering Education and Practice; 126(4), 180-185 (2000)

Palmer, S.R.; Framework for undergraduate engineering management studies, Journal of Professional Issues in Engineering Education and Practice: 129(2), 92-99 (2003)

PMI; A Guide to the Project Management Body of Knowledge (PMBOK® Guide), Third Edition (2004)

Pulko, S.H. y S. Parikh; Teaching 'soft' skills to engineers, International Journal of Electrical Engineering Education: 40( 4), 243-254 (2003)

Ribeiro, L.R.D. y M.D.N. Mizukami; Student assessment of a problem-based learning experiment in civil engineering education, Journal of Professional Issues in Engineering Education and Practice: 131(1), 13-18 (2005)

Schilling, W. y E. Hagen; Project-based learning in sanitary engineering - preliminary conclusions after three years' experience, Water Science and Technology: 41(2), 75-81 (2000) 